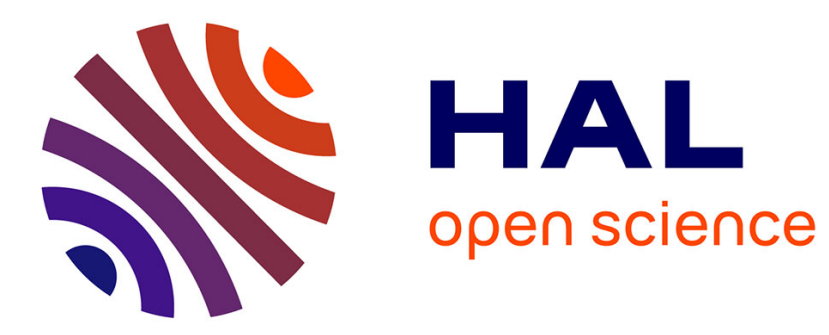

\title{
Equilibrium Signaling in Spatially Inhomogeneous Diffusion and External Forces
}

\author{
Malcolm Egan, Bayram Cevdet Akdeniz, Bao Quoc Tang
}

\section{To cite this version:}

Malcolm Egan, Bayram Cevdet Akdeniz, Bao Quoc Tang. Equilibrium Signaling in Spatially Inhomogeneous Diffusion and External Forces. IEEE Transactions on Molecular, Biological and Multi-Scale Communications, 2021, 7 (2), pp.106 - 110. 10.1109/TMBMC.2021.3054908 . hal-03099183

\section{HAL Id: hal-03099183 https://hal.science/hal-03099183}

Submitted on 6 Jan 2021

HAL is a multi-disciplinary open access archive for the deposit and dissemination of scientific research documents, whether they are published or not. The documents may come from teaching and research institutions in France or abroad, or from public or private research centers.
L'archive ouverte pluridisciplinaire HAL, est destinée au dépôt et à la diffusion de documents scientifiques de niveau recherche, publiés ou non, émanant des établissements d'enseignement et de recherche français ou étrangers, des laboratoires publics ou privés. 


\title{
Equilibrium Signaling in Spatially Inhomogeneous Diffusion and External Forces
}

\author{
Malcolm Egan, Bayram Cevdet Akdeniz and Bao Quoc Tang
}

\begin{abstract}
Complex fluid media where molecules are susceptible to forces due, for example, to external magnetic fields, complicates the design of molecular communication systems. In particular, the equations governing the motion of each molecule in time do not typically admit tractable solutions, which makes receiver design challenging for standard communication schemes; e.g., based on concentration shift keying. In this paper, a new communication scheme is proposed, which leads to simple expressions for receiver statistics, even when spatially inhomogeneous diffusion and external forces are present. The proposed scheme exploits the equilibrium statistics of the system, which arise in a wide range of scenarios. This approach is illustrated in a bounded system with inhomogeneous diffusion and external forces determined by a quadratic potential.
\end{abstract}

\section{INTRODUCTION}

A key feature of any molecular communication system is the underlying mechanism governing the motion of informationcarrying molecules in a fluid medium. For systems with a finite number of such molecules, the motion is inherently stochastic, due to random fluctuation caused by thermal processes or interactions with the molecules comprising the fluid. As such, statistical mechanics provides a sound framework to characterize the statistics of the location of each molecule [1].

There are two basic statistical models for diffusion. The first approach is known as the master equation, where the individual molecules can jump between discrete voxels [2]. The second approach is based on the Langevin stochastic differential equation, where the position of each molecule lies on a continuum [3]. Both approaches induce a Markov process, which can be described via a Fokker-Planck equation (also known as a Kolmogorov forward equation).

While the master equation approach is particularly useful for capturing both stochastic chemical kinetics and diffusion, the Smoluchowski equation arising in the Langevin approach is derived from a Newtonian perspective [4]. That is, the effect of friction and external forces on diffusion can be explicitly modeled. Indeed, the vast majority of work in molecular communications has implicitly adopted the Langevin approach in the absence of external forces, which leads to molecular motion governed by Brownian motion without drift. In some cases, drift has also been introduced, which corresponds to the assumption that information-carrying molecules are acted on by a force leading to a homogeneous velocity [1].

Key difficulties in designing molecular communication systems governed by the Smoluchowski equation are incorpo-

B. Akdeniz and M. Egan are with the CITI Lab, 69621 Villeurbanne, France which is a joint laboratory in Université de Lyon, INSA-Lyon, and INRIA. B.Q. Tang is with the Institute of Mathematics and Scientific Computing, University of Graz, Austria. rating the effect of boundary conditions, spatially inhomogeneous diffusion coefficients, and external forces. Nevertheless, particularly for sufficiently small systems with a reflective boundary, the geometry of the system has a strong impact on the motion of information-carrying molecules on short time scales [3]. On the other hand, active transport-such as in bacterial chemotaxis-is often modeled via external forces with a more complex functional form [1]. Both of these difficulties typically mean that the resulting FokkerPlanck equation-describing the probability a molecule is in a location $x$ at time $t$-does not admit a simple closed form solution, requiring further approximations or numerical methods.

As a simple expression for the probability a given molecule is observed by a receiver at a given time $t$ is not generally available, it is challenging to derive near optimal decision rules for detection at the receiver for many standard communication schemes. For example, concentration shift keying (CSK) typically requires an accurate approximation for the statistics of the number of molecules observed by the receiver before a given time $t$, often optimized to reduce the probability of error [5].

In this paper, we propose an alternative communication scheme that admits a simple approximation for the probability a molecule lies in a receiver at a sampling time. Our scheme is applicable for systems which have a finite volume (i.e., a bounded environment), a passive receiver, where inertial forces are dominated by drag forces, no degradation of informationcarrying molecules, and may be affected by an external force defined by a scalar potential as well as spatially inhomogeneous diffusion coefficients. As such, outside of special cases, it is difficult to solve the full Fokker-Planck equation to yield near optimal decision rules for the short sampling times required for standard CSK schemes.

The key idea underlying our approach is that while the full Fokker-Planck equation may be intractable, the longterm behavior of the system may be dramatically simpler. In particular, the Smoluchowski equation often converges to an equilibrium state as the sampling time $t \rightarrow \infty$ [3].

While the model in this paper accounts for diffusion-albeit in the presence of external forces-the presence of an equilibrium state bears a number of similarities with the FokkerPlanck equation arising from the reaction-diffusion master equation (RDME) accounting for chemical reactions and a simplified model of diffusion [6]. Recently, we developed an analogous communication scheme for RDME models [2], valid for non-trivial geometries but without external forces, and we show that the same detection algorithm can be applied to the Langevin model considered in this paper. 
To illustrate our approach, we consider a system with a quadratic potential and inhomogeneous diffusion coefficients, which can, for example, arise due to the presence of electrostatic steering of molecules [7]. While deriving the optimal sampling time and the resulting detection rule requires extensive numerical computations in the case of standard CSK schemes, the equilibrium distribution admits a very simple form. We show that even for finite sampling times, it is feasible to obtain a good approximation for the receiver statistics and use this to obtain a near optimal decision rule for sufficiently long symbol periods.

\section{SySTEM MODEL}

Let $\Omega=\left[b_{l}, b_{r}\right], \quad-\infty<b_{l}<b_{r}<\infty$ be a bounded onedimensional domain consisting of transmitting and receiving devices, with a fluid medium separating the devices. Messages to be sent by the transmitter are encoded into the quantity of molecules of a chemical species $S$, which diffuses within the domain $\Omega$. The receiver domain is denoted by $\Omega_{\mathrm{Rx}}$.

Consider a single information-carrying molecule, which is colloidal; i.e., larger than the liquid molecules forming the fluid medium. In this paper, we assume that the motion of each molecule is independent and governed by the Smoluchowski equation [3]

$$
\gamma(x) \frac{\mathrm{d} x}{\mathrm{~d} t}=F(x)+\sigma(x) \xi(t),
$$

where $x=x(t) \in \mathbb{R}$ is the location of the molecule at time $t, \xi(t)$ is a standard Wiener process, $\gamma(x)>0$ is a friction constant, $\sigma(x)>0$ is the scale parameter of the noise, and $F$ is an external force.

Associated with the Smoluchowski equation in (1) is a Fokker-Planck equation, given by [3]

$$
\begin{aligned}
\frac{\partial}{\partial t} p\left(x, t \mid x_{0}, t_{0}\right)= & \frac{\partial^{2}}{\partial x^{2}}\left(D(x) p\left(x, t \mid x_{0}, t_{0}\right)\right) \\
& -\frac{\partial}{\partial x}\left(\frac{F(x)}{\gamma(x)} p\left(x, t \mid x_{0}, t_{0}\right)\right),
\end{aligned}
$$

where $p\left(x, t \mid x_{0}, t_{0}\right)$ is the probability density function for the location $x$ of the molecule at time $t$, given an initial location $x_{0}$ at time $t_{0}$, and $D(x)=\frac{\sigma(x)^{2}}{2 \gamma(x)^{2}}$ is the diffusion coefficient. Note that both the Smoluchowski equation in (1) and the FokkerPlanck equation are equivalent descriptions of the system dynamics. Moreover, in general, the diffusion coefficient is spatially inhomogeneous; i.e., the diffusion coefficient depends on the location $x$. In the special case where there are no external forces and the diffusion coefficient, $D$, is constant, the Fokker-Planck equation for Brownian motion (commonly called Fick's law) is obtained as

$$
\frac{\partial}{\partial t} p\left(x, t \mid x_{0}, t_{0}\right)=D \frac{\partial^{2}}{\partial x^{2}} p\left(x, t \mid x_{0}, t_{0}\right) \text {. }
$$

To account for the finite volume of the system, it is necessary to also introduce boundary conditions. In the case of reflective boundaries, considered in this paper, the boundary conditions are given by

$$
\begin{aligned}
& j\left(b_{l}, t \mid x_{0}, t_{0}\right)=0 \\
& j\left(b_{r}, t \mid x_{0}, t_{0}\right)=0,
\end{aligned}
$$

where

$$
j\left(x, t \mid x_{0}, t_{0}\right)=\frac{\partial}{\partial x}\left(D(x) p\left(x, t \mid x_{0}, t_{0}\right)\right)-\frac{F(x)}{\gamma(x)} p\left(x, t \mid x_{0}, t_{0}\right)
$$

is the flux at location $x$.

Under certain conditions detailed in the following section, the Fokker-Planck equation in (2) admits an equilibrium state. That is, the solution $p_{\infty}(x)$ satisfies the condition that the flux vanishes; i.e.,

$$
j\left(x, t \mid x_{0}, t_{0}\right)=0 .
$$

Moreover, the form of $p_{\infty}$ is sufficiently tractable to develop near optimal detection rules for sufficiently long symbol periods. In the following section, we develop a new signaling and detection scheme exploiting this property.

\section{Signaling AND DETECTION}

In this section, we detail our proposed scheme. We focus on the case of binary signaling with equally likely symbols. That is, for the transmitter to send a bit 1 , it generates $\Delta$ molecules of species $S$ within the transmitter. For the case of bit 0 , the transmitter generates zero molecules of S. As we will show, the precise locations where the molecules of $S$ are generated within the transmitter does not affect receiver design, due to the fact that the equilibrium solution remains the same.

\section{A. Communication Scheme}

Assume that the system operates using time slots with duration $T_{s}$ and that no molecules of $\mathrm{S}$ are present in the system at time $t=0$. The bit to be transmitted in time slot $n$ is denoted by $s_{n}$. Molecules of $S$ may then diffuse throughout the system; however, no molecules degrade.

Consider the $n$-th time slot. Due to the previous $n-1$ transmissions, there are $N_{\mathrm{Tx}}\left(n T_{s}\right)$ molecules of species $\mathrm{S}$ in the transmitter. At a time $n T_{s}+\delta$ shortly after the beginning of the $n$-th time slot, transmitter produces a quantity of $\mathrm{S}$ depending on the bit to be transmitted. In particular,

$$
N_{\mathrm{Tx}}\left(n T_{s}+\delta\right)=\left\{\begin{array}{cc}
N_{\mathrm{Tx}}\left(n T_{s}\right)+\Delta & s_{n}=1, \\
N_{\mathrm{Tx}}\left(n T_{s}\right) & s_{n}=0,
\end{array}\right.
$$

for $\delta>0$ a sufficiently small period of time; that is, $\delta$ is chosen such that no molecules of $S$ diffuse outside of the transmitter.

The key idea behind the proposed communication scheme is that for sufficiently large $T_{s}$, the total number of molecules of $\mathrm{S}$ will be approximately drawn from the stationary distribution of the Fokker-Planck equation. As such, since the equilibrium statistics are known, near-optimal detection rules can be derived.

\section{B. Equilibrium Statistics}

In the presence of reflective boundary conditions, the Fokker-Planck equation admits an equilibrium solution if the fluctuation-dissipation condition is satisfied and the external force is determined by a scalar potential. That is, in the presence of a scalar potential, a sufficient condition for an equilibrium solution to exist is for the fluctuation-dissipation condition to hold. 
In particular, a general form of the fluctuation-dissipation condition $^{1}$ accounting for spatially inhomogeneous diffusion is given by [9]

$$
\frac{\partial}{\partial x} D(x)=F(x)\left(\gamma(x)^{-1}-D(x) \beta\right),
$$

where $\beta=\frac{1}{k_{B} T}, k_{B}$ is Boltzmann's constant and $T$ is the temperature of the system.

In this case, the Fokker-Planck equation in (2) can be written as

$$
\begin{aligned}
\frac{\partial}{\partial t} p\left(x, t \mid x_{0}, t_{0}\right)= & \frac{\partial}{\partial x}\left(D(x) \frac{\partial}{\partial x} p\left(x, t \mid x_{0}, t_{0}\right)\right) \\
& -\frac{\partial}{\partial x} \beta F(x) D(x) p\left(x, t \mid x_{0}, t_{0}\right) .
\end{aligned}
$$

If the external force is determined by a scalar potential function $U$, the force satisfies $F=-\frac{\partial U}{\partial x}$ and $U$ is bounded in $\left[b_{l}, b_{r}\right]$, then the equilibrium solution to the Fokker-Planck equation in (2) is given by $[3, \mathrm{Sec} .5 .2]$

$$
p_{\infty}(x)=Z e^{-\beta U(x)},
$$

where $Z=\left(\int_{b_{l}}^{b_{r}} e^{-\beta U(x)} \mathrm{d} x\right)^{-1}$ is the normalizing constant. Note that scalar potentials are ubiquitous; for example, arising from electrostatic and gravitational fields. We also highlight that the equilibrium solution $p_{\infty}$ in (10) is remarkable in that it only depends on $\beta$ and the potential $U$. That is, given these parameters, it is straightforward to obtain the asymptotic statistics of the system. This is true even if the diffusion coefficient is spatially inhomogeneous.

The equilibrium solution to the Fokker-Planck equation provides information about the statistics for the location of a single molecule. The probability that the molecule lies in the receiver at a sufficiently large sampling time $T_{s}$ can then be well approximated by

$$
p_{\mathrm{Rx}}\left(T_{s}\right) \approx \int_{\Omega_{\mathrm{Rx}}} p_{\infty}(x) \mathrm{d} x=\int_{\Omega_{\mathrm{Rx}}} Z e^{-\beta U(x)} \mathrm{d} x .
$$

To derive the statistics for the quantity of molecules in the receiver at the sampling time $T_{s}$, let $X_{i}$ be a Bernoulli random variable with success probability $p_{\mathrm{Rx}}\left(T_{s}\right)$, which indicates whether or not molecule $i$ lies in the receiver. Suppose that $\Delta$ molecules have been emitted into the system before a sampling time $n T_{s}$. The total number of molecules in the receiver at the sampling time is then given by

$$
X_{t o t}=\sum_{i=1}^{\Delta} X_{i}
$$

Since each molecule's motion is assumed to be independent, for a sufficiently large $\Delta$, it follows from the central limit theorem that $X_{t o t}$ is then well approximated by a Gaussian random variable

$$
\tilde{X}_{t o t} \sim \mathcal{N}\left(\Delta p_{\mathrm{Rx}}\left(n T_{s}\right), \Delta p_{\mathrm{Rx}}\left(n T_{s}\right)\right) .
$$

for $p_{\mathrm{Rx}}\left(n T_{s}\right)$ sufficiently small.

\footnotetext{
${ }^{1}$ The reader is referred to [8] for a general discussion of fluctuationdissipation conditions.
}

\section{Near-Optimal Detection}

Under the assumption that the statistics for the number of molecules in the receiver at a sampling time $n T_{s}$ given $\Delta$ molecules are present in the system at time $(n-1) T_{s}+\delta$ is given by (13), it is now feasible to derive detection rules. In particular, we seek to obtain an estimate for the transmitted sequence $\left(s_{1}, \ldots, s_{n+1}\right) \in\{0,1\}^{n+1}$. Although the observation process is Markovian, for a sufficiently large time slot $T_{s}$, the observations $\mathbf{N}_{\mathrm{Rx}}=\left(N_{\mathrm{Rx}}\left(T_{s}\right), \ldots, N_{\mathrm{Rx}}\left((n+1) T_{s}\right)\right)$ at each sampling time $k T_{s}, k=1,2, \ldots$ are approximately independent.

Let $\mathbf{s} \in\{0,1\}^{n+1}$ denote a potential vector of transmitted bits. Under the Gaussian approximation in (13), the joint likelihood of the observations is given by

$$
\begin{aligned}
& f_{\mathbf{N}_{\mathrm{Rx}} \mid \mathbf{s}}(\mathbf{n}) \\
& =\prod_{i=1}^{n+1} \frac{1}{\sqrt{2 \pi \mu_{r} \sum_{j=1}^{i} s_{j}}} \exp \left(-\frac{\left(n_{i}-\mu_{r} \sum_{j=1}^{i} s_{j}\right)^{2}}{2 \mu_{r} \sum_{j=1}^{i} s_{j}}\right),
\end{aligned}
$$

where $\mu_{r}=p_{\mathrm{Rx}}\left(T_{s}\right) \Delta$, with $\Delta$ as in (7). Moreover, assuming the independence of elements of $\mathbf{N}_{\mathrm{Rx}}$ and the validity of (14), the optimal detection rule is given by

$$
\hat{\mathbf{s}}^{*}=\arg \max _{\mathbf{s} \in\{0,1\}^{n+1}} f_{\mathbf{N}_{\mathrm{Rx}} \mid \mathbf{s}}(\mathbf{n}) .
$$

A form of the Viterbi algorithm with appropriate branch weights can be used to efficiently solve the optimization problem in (15). The algorithm is detailed in Algorithm 1. For the $k$-th symbol $s_{k} \in\{0,1\}$, let $p\left(n_{k} \mid s_{k}\right)=$ $\log \left(f_{N_{\mathrm{Rx}, 1}\left(k T_{s}\right) \mid \mathbf{s}_{k}}\left(n_{k}\right)\right)$, where $\mathbf{s}_{k}$ are the symbols in $\mathbf{s}$ up to time $k T_{s}$. In the $k$-th symbol interval, it is necessary to compute $P_{k-1,0}$ and $P_{k-1,1}$, which correspond to the probability of the most probable sequence until the $(k-1)$-th symbol is 0 and 1 , respectively.

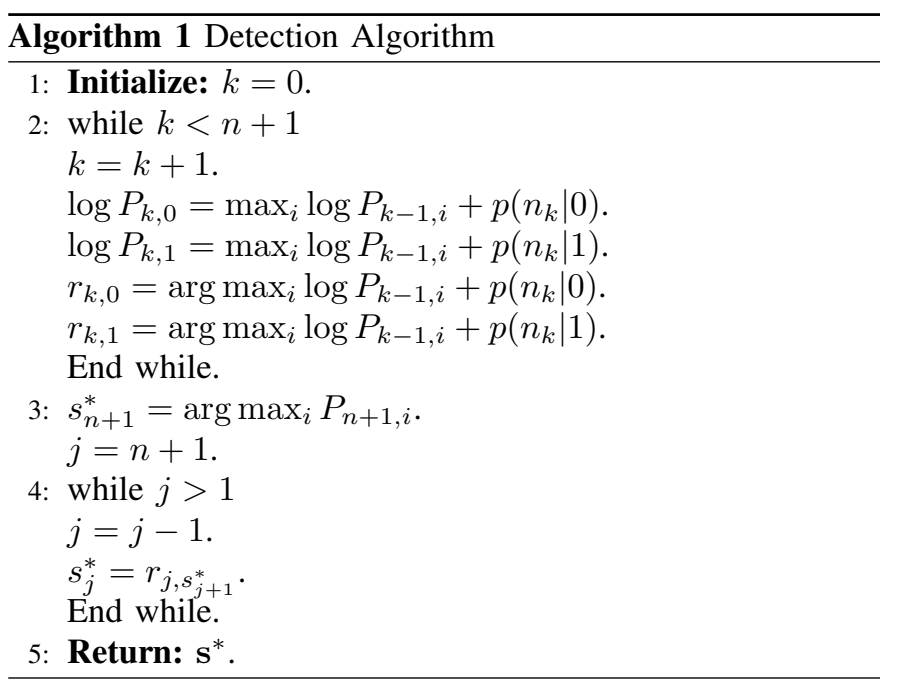

We remark that Algorithm 1 has the same form as the near optimal detection algorithm developed in [2] for equilibrium signaling in the presence of chemical reactions. The reason for this is that both systems converge to an equilibrium inducing receiver observations with known approximately Gaussian statistics. 


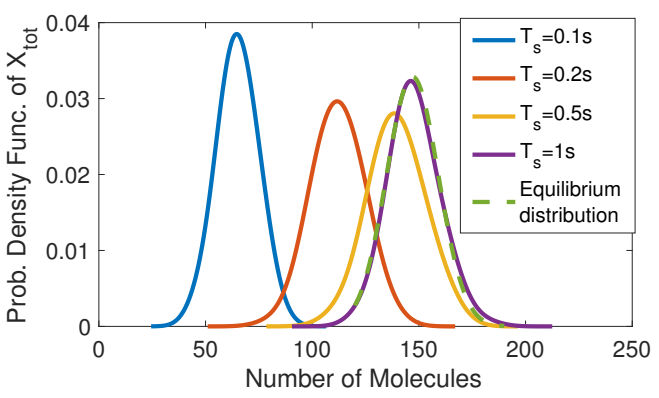

Fig. 1. Verification of (13) for varying $T_{s}$, with $\Delta=1000$ molecules, $D_{0}=10^{-11} \mathrm{~m}^{2} / \mathrm{s}, \kappa=10^{-9} \mathrm{~N} / \mathrm{m}, \beta=\frac{1}{4.11 \times 10^{-21}} \mathrm{~J}, \alpha=0.1$.

\section{NumERICAL RESULTS}

In this section, we illustrate our communication scheme in the presence of spatially inhomogeneous diffusion and a quadratic external potential, which acts by pushing molecules towards the center of the channel. We note that this scenario is challenging for standard CSK schemes due to the fact that the finite time behavior of the system must be characterized. In particular, as the Fokker-Planck equation does not admit tractable solutions for finite times, detection rules are difficult to obtain.

We assume that the system lies in the domain $[-4 \mu \mathrm{m}, 4 \mu \mathrm{m}]$, the transmitter is placed at $x=-1 \mu \mathrm{m}$ and the receiver is defined by the domain $[1 \mu \mathrm{m}, 2 \mu \mathrm{m}]$. The diffusion coefficient is assumed to be $D(x)=D_{0} e^{-\alpha x^{2}}$, with $D_{0}, \alpha>0$, which is spatially inhomogeneous due to the dependence of the diffusion coefficient on $x$. Note that larger $D_{0}$ and smaller $\alpha$ increase the rate of convergence to equilibrium. We assume that molecules are influenced by a quadratic external potential leading to a force $F=-\kappa|x|, \kappa>0$. The friction and scale parameters, $\gamma$ and $\sigma$ respectively, in (2) are then determined via the fluctuation-dissipation condition in (8), which ensures that the system converges to an equilibrium state.

In order to obtain a solution of (2), we utilize the EulerMaruyama method, detailed in [10]. To account for the reflective boundary conditions, when a molecule passes the boundary, it is projected into the domain $[-4 \mu \mathrm{m}, 4 \mu \mathrm{m}]$ as detailed in [11]. Unless otherwise stated, parameters are chosen as $D_{0}=10^{-11} \mathrm{~m}^{2} / \mathrm{s}, \kappa=10^{-9} \mathrm{~N} / \mathrm{m}, \beta=$ $\frac{1}{4.11 \times 10^{-21}} J, \alpha=0.1$.

Due to the fact that we require a finite sampling time, a key question is how small the symbol period can be while still yielding a good approximation of the equilibrium distribution. Fig. 1 plots the empirical estimate of the distribution for the number of molecules with different symbol periods. The distribution is estimated via kernel density estimation with bandwidth parameter $h=5$. Observe that the receiver statistics rapidly converge to the distribution expected from (13).

In Fig. 2, we plot the average probability of error as defined in [2, Eq. (36)] for varying quantity of molecules $\Delta$. In particular, we have calculated the probability of error for the communication channel defined in Section III by transmitting 100 consecutive bits and repeating this process for 5000 iterations. Observe that the probability of error rapidly

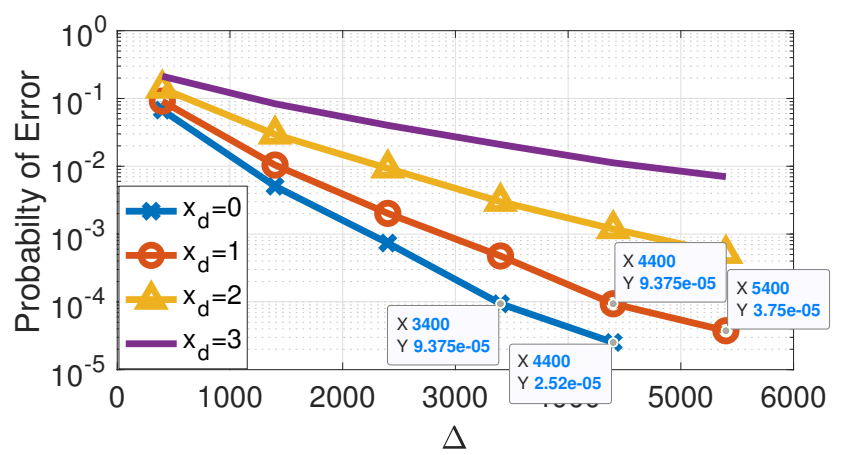

Fig. 2. Average probability of error with different initial position, $x_{d} \mu \mathrm{m}$, of the receiver with a region $\left[x_{d} \mu \mathrm{m}, x_{d}+1 \mu \mathrm{m}\right]$ and varying quantity of molecules $\Delta$.

decays, suggesting that the detection rule is well adapted to the receiver statistics. We also see that as the distance between the transmitter and receiver increases, corresponding to varying $x_{d}$, the probability of error increases, which is a consequence of the external force applied to each molecule. In particular, the external force concentrates the probability density function for the location of a single molecule towards the center of the domain.

\section{CONCLUSION}

To develop molecular communication strategies in complex environments, it is necessary to have accurate characterizations of the statistics for the number of molecules within the receiver. In this paper, we proposed a new method to derive near optimal detection rules for sufficiently large symbol periods. Numerical results validated our approach in the presence of a force governed by a quadratic potential and spatially inhomogeneous diffusion.

\section{REFERENCES}

[1] I. Akyildiz, M. Pierobon, and S. Balasubramaniam, "An information theoretic framework to analyze communication systems based on statistical mechanics," Proceedings of the IEEE, vol. 107, no. 7, pp. 1230-1255, 2019.

[2] B. Akdeniz, M. Egan, and B. Tang, "Equilibrium signaling: molecular communication robust to geometry uncertainties," IEEE Transactions on Communications, early access. doi:10.1109/TCOMM.2020.3034662, pp. 1-1, 2020.

[3] H. Risken, The Fokker-Planck Equation: Methods of Solution and Applications. Springer, 1989.

[4] W. Coffey, Y. Kalmykov, and J. Waldron, The Langevin Equation. World Scientific, 2004.

[5] N. Farsad, H. B. Yilmaz, A. Eckford, C.-B. Chae, and W. Guo, "A comprehensive survey of recent advancements in molecular communication," IEEE Communications Surveys \& Tutorials, vol. 18, no. 3, pp. 18871919, 2016

[6] D. Gillespie, "The chemical Langevin equation," The Journal of Chemical Physics, vol. 113, no. 1, pp. 297-306, 2000.

[7] R. Wade, R. Gabdoulline, S. Lüdemann, and V. Lounnas, "Electrostatic steering and ionic tethering in enzyme-ligand binding: insights from simulations," Proc. Nat. Acad. Sci., vol. 95, pp. 5942-5949, 1998.

[8] P. Grassia, "Dissipation, fluctuations, and conservation laws," American Journal of Physics, vol. 69, no. 2, pp. 113-119, 2001.

[9] K. Schulten and I. Kosztin, Lectures in Theoretical Biophysics. University of Illinois, 2000.

[10] D. J. Higham, "An algorithmic introduction to numerical simulation of stochastic differential equations," SIAM review, vol. 43, no. 3, pp. 525546, 2001.

[11] D. Lépingle, "Euler scheme for reflected stochastic differential equations," Mathematics and Computers in Simulation, vol. 38, no. 1-3, pp. 119-126, 1995. 


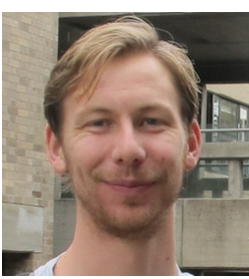

Malcolm Egan (S'10-M'14) received the Ph.D. in Electrical Engineering in 2014 from the University of Sydney, Australia. He is currently a Chargé de Recherche (Permanent Research Staff) in Inria hosted by CITI, a joint laboratory between Inria, INSA Lyon and Université de Lyon, France. Previously he was a Assistant Professor in INSA Lyon, and a Postdoctoral Researcher in the Laboratoire de Mathématiques, Université Blaise Pascal, France and the Department of Computer Science, Czech Technical University in Prague, Czech Republic. $\mathrm{He}$ is currently an Associate Editor for IEEE Communications Letters and previously a guest editor for IEEE Access. His research interests include are in the areas of information theory and statistical signal processing with applications in wireless and molecular communications.

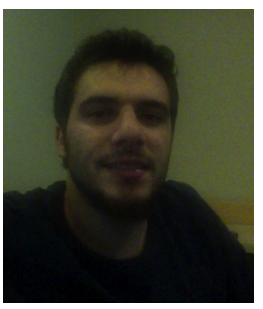

Bayram Cevdet Akdeniz received his B.S. degree in Electronics and Communication engineering in Yildz Technical University, and the M.Sc. and Ph.D degrees in Electrical-Electronics engineering from Bogazici University, Istanbul, Turkey, in 2010, 2013 and 2018 respectively. He worked as a post-doctoral researcher in Inria hosted by CITI, a joint laboratory between Inria, INSA Lyon and Université de Lyon, France between 2018 and 2020 . He is currently a post-doctoral researcher at Centre for Bioinformatics at University of Oslo. His research interests include, Molecular Communication, Nanonetworks, Communication theory Bioinformatics and Machine Learning.

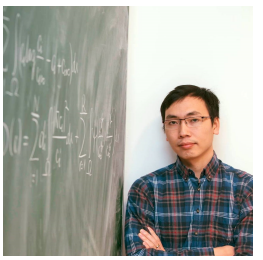

Bao Quoc Tang received his Ph.D. in Mathematics in 2015 from the University of Graz, Austria. Currently, he is a University Assistant (on par with nontenure track Assitant Professor) at the Institute for Mathematics and Scientific Computing, University of Graz. His research includes reaction-diffusion systems arising from chemistry, biology, or physics, and their applications to molecular communications as well as to game theory. 